\title{
Memory Assessment Battery and Early Identification of Dementia
}

\author{
F. Ostrosky-Solís, Y. Rodríguez, C. García de la Cadena, R.M. Jaime, \\ A.M. Valdés, R. Chayo-Dichi, D. Velázquez, M. Pérez, M. Guevara I. Madrazo* and C. Cuevas \\ Department of Psychophysiology, Graduate School, National University of Mexico, \\ *Research Unit, Centro Médico Siglo XXI, Mexico
}

Early identification of dementia is of great importance for the development of any potential therapy that attempts to prevent, delay or alter the progression of the disease. Furthermore, any potential treatment must be offered during early stages of the demential processes since it is during these stages that treatment is most likely to be of benefit. Effective assessment of treatment requires the use of reliable and sensitive measurements of the target functions. A prominent sign of dementia of the Alzheimer's type (DAT) is a deficit in the ability to learn and remember new information. A battery of memory functions was established including: a) neuropsychological measures of memory (immediate and delayed recall; learning; cued recall; and recognition for verbal and non-verbal material for auditory, visual and spatial information); b) electrophysiological measures linked to memory processes (late-event related potentials-P300 paradigm), and c) functional measures of everyday memory. The battery of tests was applied to 66 healthy volunteers between 20 and 100 years of age. Subjects were divided into four age groups: 20-39, 40-59, 60-75, 76-100. Results showed that during aging not all aspects of memory and learning are equally affected. Some tests appeared particularly sensitive to the effects of aging (such as delayed recall in the visual and auditory modality for verbal and nonverbal information). For others, the effects of aging were minimally observed or not revealed at all (such as immediate memory for auditory, visual and spatial information). Electrophysiological measures showed that P300 latency increased in direct relation with age. The P300 reached its peak later in the elder subjects than in the younger subjects. The amplitude of the P300 did not change with age. A principal component analysis with all the neuropsychological and electrophysiological variables was applied. Four components accounted for $63.4 \%$ of the total variance. Factor 1 with an eigenvalue of 10.07 explained $37.1 \%$ of the variance and grouped the neuropsychological measures of memory which required storage and recall of information as well as the latency of the P 300 . These data suggest that the P300 component is related more to storage and retrieval of information than to the early stages of information processing. Our battery assesses several aspects of memory, and some tests appeared particularly sensitive to the effects of aging, whereas in others the effects of aging were minimal. These data reflect the involvement of different underlying neural systems. Neuropsychological and electrophysiological measures which do not decline with age can be as useful in the diagnosis of dementia as performance on tests which are sensitive to the effects of normal aging, since failure on these tests would indicate abnormal pathological aging. Normal aging data will provide a reference for differential diagnosis and early identification of $A D$, as well as for the evaluation of any potential treatment that might attempt to alter the early symptoms of this disease.

This study was partially supported by a grant from CONACYT Number 0772-H0109. 

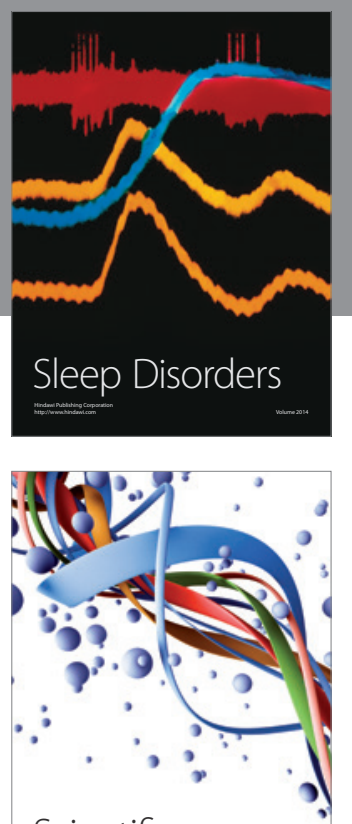

Scientifica
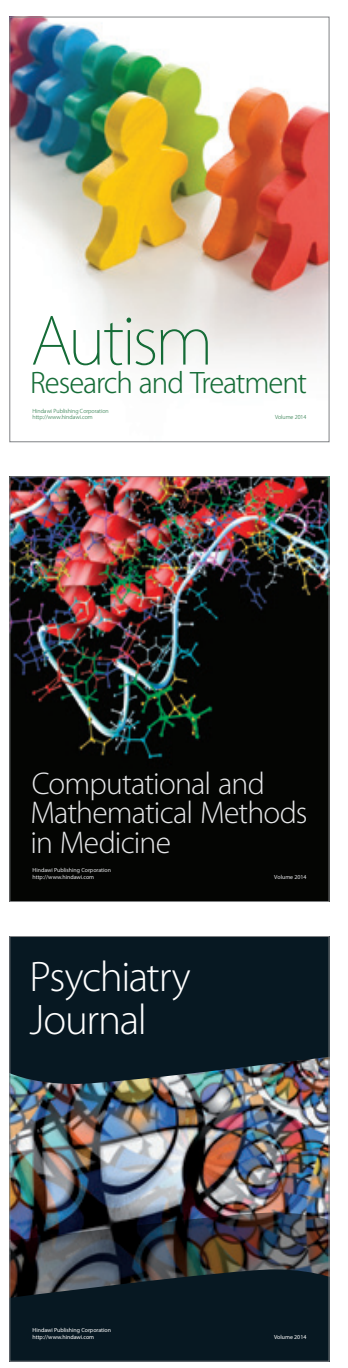
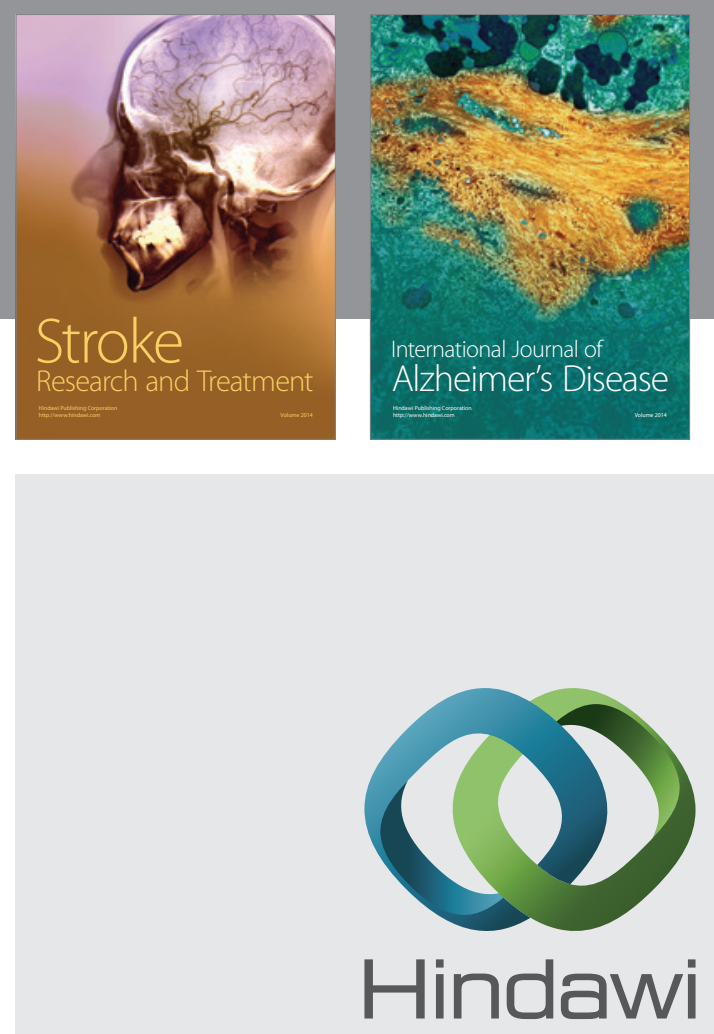

Submit your manuscripts at

http://www.hindawi.com
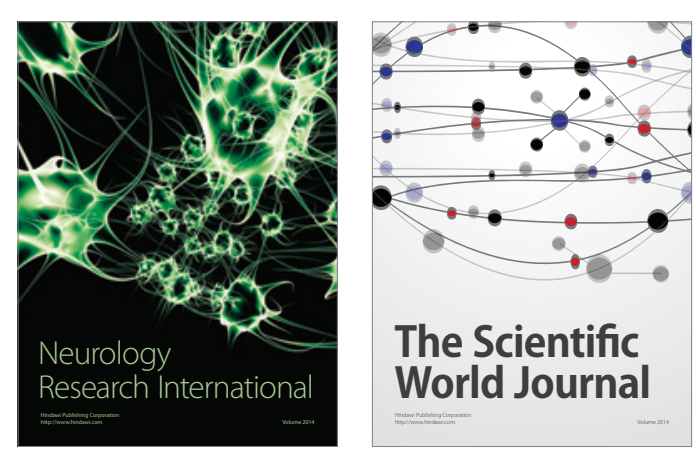

The Scientific World Journal

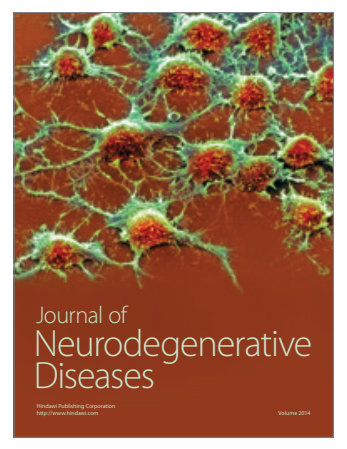

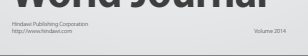

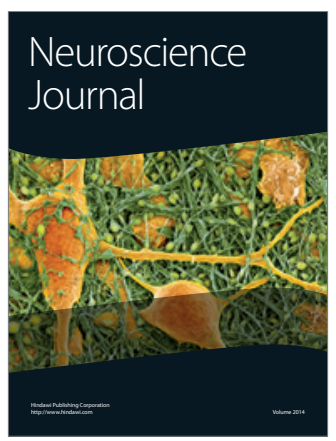

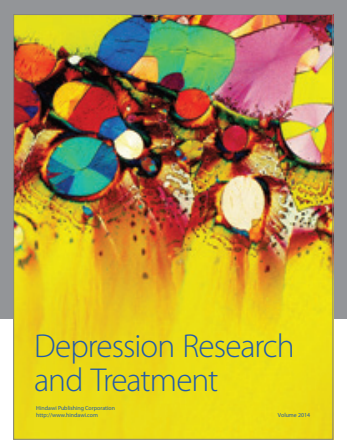
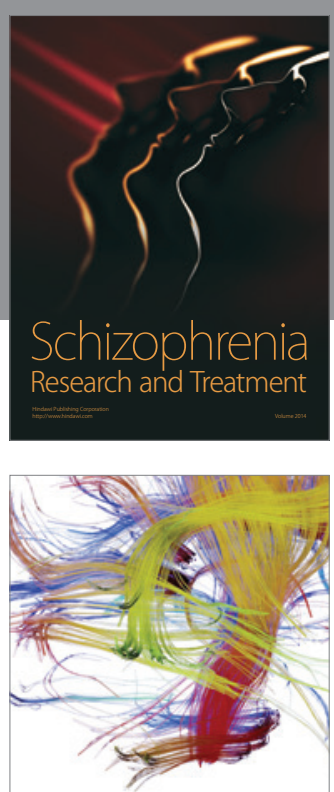

Brain Science

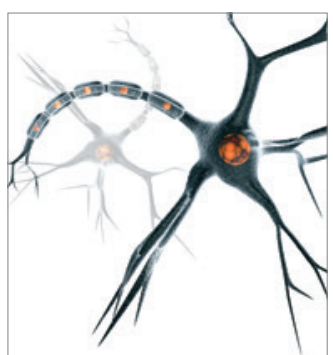

Neural Plasticity
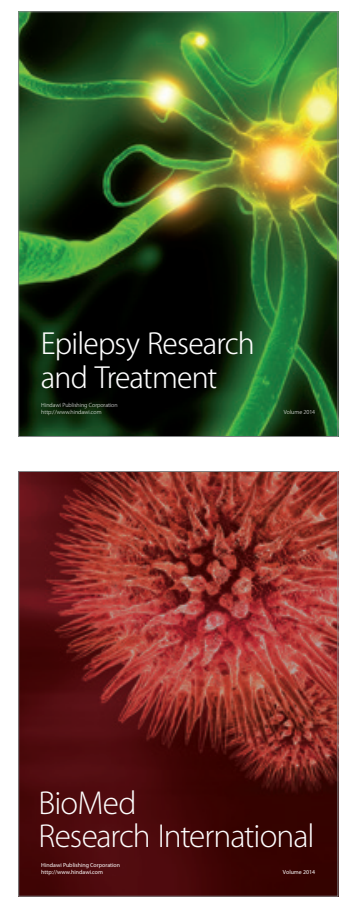

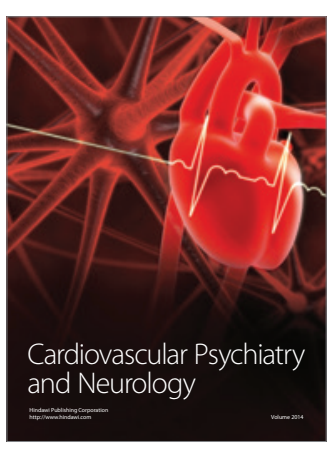

Parkinson's

Disease
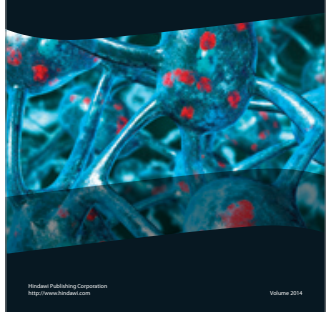